\title{
Abnormal Visual Adaptation to Flicker in Multiple Sclerosis
}

\author{
J.E. Raymond
}

\begin{abstract}
A visual psychophysical adaptation procedure was used on patients with Multiple Sclerosis (MS) in an attempt to induce a temporary and local exacerbation of subclinical visual impairment. Using a flicker detection task, sensitivity before and after adaptation to a flickering stimulus was measured in 9 MS patients and 9 control subjects. Although only $22 \%$ of patient eyes had abnormal flicker sensitivity prior to adaptation, visual deficit was observed in $83 \%$ of eyes studied after adaptation. Of the $7 \mathrm{MS}$ eyes studied for which no other sign or symptom of visual involvement was present, 5 were found to have visual deficits after flicker adaptation. In addition, 10 of the 11 eyes affected by MS showed an abnormal response to flicker adaptation. Recovery from the effects of adaptation was complete in all patients within 2 minutes. The results suggest that partial demyelination of visual pathway neurons may exist in patients without signs or symptoms of visual involvement. The prolonged stimulation provided during adaptation may produce a temporary fatiguing or conduction blockade of such neurons which may lead to reductions in sensory sensitivity.
\end{abstract}

\begin{abstract}
RÉSUMÉ: Adaptation visuelle anormale à la stimulation lumineuse intermittente dans la sclérose en plaques Nous avons utilisé une technique d'adaptation visuelle psychophysique chez des patients atteints de sclérose en plaques (SEP) dans le but d'induire une exacerbation temporaire et locale de l'atteinte visuelle subclinique. A l'aide d'une épreuve de détection du papillotement, nous avons mesuré la sensibilité avant et après adaptation à une stimulation lumineuse intermittente chez 9 patients atteints de SEP et 9 sujets contrôles. Même si seulement $22 \%$ des yeux des patients avaient une sensibilité anormale à la stimulation lumineuse intermittente avant adaptation, un déficit visuel a été observé chez $83 \%$ des yeux étudiés après adaptation. Parmi les 7 yeux SEP étudiés qui ne manifestaient aucun signe ou symptome d'atteinte visuelle, 5 présentaient des déficits visuels après adaptation à la stimulation lumineuse intermittente. De plus, 10 des 11 yeux atteints de SEP présentaient une réponse d'adaptation anormale. La récupération des effets de l'adaptation était complète chez tous les patients en dedans de 2 minutes. Ces résultats suggèrent qu'une démyélinisation partielle des neurones des voies de transmission de l'influx visuel peut exister chez des patients ne manifestant aucun signe ou symptome d'atteinte visuelle. La stimulation prolongée fournie pendant l'adaptation peut produire une fatigue temporaire ou un blocage de la conduction de tels neurones, pouvant induire une diminution de la sensibilité sensorielle.
\end{abstract}

Can. J. Neurol. Sci. 1988; 15:286-291

Extensive demyelination of neurons in the primary visual pathways is almost universally found in patients with advanced multiple sclerosis (MS) who are examined at autopsy, 1,2 However, investigations of visual disorders in MS patients have revealed abnormalities in only 30 per cent of patients when conventional testing procedures (e.g., Snellen acuity, static perimetry, fundoscopic examination) are used. ${ }^{3}$ In view of these findings, it is likely that visual system pathology is present in a considerable proportion of MS patients during life but that it is not always manifested as clinically evident fundoscopic changes or as obvious disturbances in visual function. Recently, psychophysical techniques for measuring visual function have been used experimentally as a means of detecting subtle visual disturbances in MS patients.

One class of such visual tests have employed temporal visual discriminations in an effort to demonstrate abnormally slowed or delayed neural transmission in the visual pathways of MS patients. For example, in double flash 4 and multi-flash 5 campimetry, subjects view a test spot at various locations in the visual field and are asked to judge whether they perceive a single illumination of a test spot or multiple successive flashes. The duration between successive flashes of light is lengthened until the subject reports the perception of flicker. Using these techniques, it was found that a large proportion of MS patients with a history of optic neuritis could tolerate substantially longer inter-stimulus intervals before flicker perception was reported than healthy control subjects. These results are consistent with neurophysiological data on experimentally demyelinated neurons which suggest that the temporal order of neural signals transmitted by demyelinated axons is under considerable temporal disarray. However, abnormal multi-flash campimetry results may also be found in patients with amblyopia, cataract, macular degeneration and optic neuritis not associated with demyelinating disease. ${ }^{6}$

From the Department of Psychology, University of Calgary.

Received November 5, 1987. Accepted in final form April 23, 1988

Reprint requests to: Dr. J. Raymond, Department of Psychology, University of Calgary, 2500 University Drive, Calgary, Alberta T2N 1N4 Canada. 
Other tests based on flicker perception which have reported abnormal visual function in MS include techniques which assess critical flicker fusion frequency, 7,8 the Pulfrich illusion, ${ }^{9}$ differences of visual latencies in the two eyes, 10 DeLange functions, 11 temporal contrast sensitivity functions 12 and temporal frequency discrimination functions. ${ }^{13}$ In all of these reports, the MS patient samples consisted of individuals with previously known visual signs or symptoms. Thus, while these psychophysical tests have proved successful at detecting visual system involvement in substantial proportions of carefully selected patients, it is not known whether they are successful at revealing visual system damage in patients with subclinical visual involvement.

In the earlier stages of MS, when diagnosis is a critical issue, lesions may be too small to result in measurable deficits of visual function. Techniques which could temporarily exacerbate the effect of such lesions so as to render symptoms psychophysically observable could be potentially useful as diagnostic procedures. For example small elevations in body temperature ${ }^{14}$ or prolonged physical exercise ${ }^{15}$ has been reported to produce temporary deficits in vision in MS patients.

Research on demyelination in animals has shown that a temporary increase in the probability of complete axonal conduction blockade can also be produced by stimulation of a partially demyelinated neuron with high frequency trains of electrical impulses. ${ }^{16,17}$ This suggests that excessive stimulation of visual neural units through prolonged exposure to specific suprathreshold visual stimuli (i.e., adaptation) might increase conduction blockade in MS patients, thus leading to a temporary worsening of visual symptoms. Unlike elevation in body temperature, the temporary exacerbation of symptoms would be local to the visual system and not cause a generalized discomfort to the patient.

Visual adaptation (e.g., to high contrast grating patterns) has been shown to produce small transient changes in visual (e.g., contrast) thresholds obtained from healthy subjects (e.g., 18). These well-studied psychophysical adaptation effects demonstrate the impact prolonged exposure to specific stimuli has on the healthy visual system and have been useful for gaining an understanding of visual mechanisms. If the visual pathways of MS patients are only partially demyelinated, then visual adaptation procedures may be particularly successful at revealing the visual pathology that is undetectable using conventional techniques. Given the physiological data on the effects of demyelination on neural transmission and the psychophysical data on flicker perception abnormalities in MS patients, adaptation tech- niques involving temporal discriminations may be particularly successful at revealing subtle visual disturbance in MS.

Using healthy observers, Smith ${ }^{19}$ reported that after prolonged viewing of a highly modulated flickering disk, the minimum depth of modulation needed to detect flicker of the same frequency as the adapting stimulus was elevated relative to that required before adaptation. The present experiment investigated this adaptation effect in MS patients. The results indicate that relative to healthy controls, MS patients undergo abnormally rapid and large losses in flicker sensitivity during adaptation.

\section{MeThodS}

\section{Patients and Control Subjects}

The control group consisted of 5 females and 4 males chosen from university students and staff whose ages ranged from 20 to 28 years (mean $=23.1$ years). All subjects had 20/30 acuity or better in both eyes and no history of neurological disorder. Control subjects were paid an hourly fee. The MS group wats composed of 9 patient volunteers ( 8 females and 1 male) ranging in age from 21 to 53 years (mean $=36.3$ ). All patients were diagnosed "definite" MS according to the criteria set out by Schumacher et al. ${ }^{20}$ and were rated on the disability status scale of Kurtzke. ${ }^{21}$ Clinical data for the patients are listed in Table !. All patients had good manual dexterity and experienced no difficulty manipulating the knob used to control flicker in the display.

Patients were assessed for visual acuity, optic disk colour, pupillary defects and, in most cases, contrast sensitivity and double flash sensitivity. Two of the nine patients had no sign or symptom of visual involvement in either eye. Three patients had evidence of monocular involvement and the remaining four showed bilateral involvement. None of the patients were on medication at the time of the experiment.

\section{Apparatus and Stimuli}

Flickering stimuli were generated by light emitting diodes (LEDs) driven by custom electronics. A single Wein bridge electronic oscillator sinusoidally modulated the luminance of both the adapting and test stimuli. LEDs were used because they have a rapid response to input current. A tightly packed matrix of 13 LEDs attached to the end of a $105 \mathrm{~cm}$ long mirror box and viewed through a $2 \mathrm{~mm}$ thick milk glass diffuser was used to produce the adapting field display. When viewed by the subject, a spatially uniform red (approximately $650 \mathrm{~nm}$ ) circular field $2.5 \mathrm{deg}$ in diameter was seen. During adaptation, the lumi-

\begin{tabular}{|c|c|c|c|c|c|c|}
\hline Age & Sex & $\begin{array}{c}\text { Kurtzke } \\
\text { Scale }\end{array}$ & Visual Indications & $\begin{array}{c}\text { Years Since } \\
\text { Onset }\end{array}$ & $\begin{array}{c}\text { Snellen } \\
\mathbf{L}\end{array}$ & $\underset{\mathbf{R}}{\text { Acuity }}$ \\
\hline 23 & $\mathrm{f}$ & 2 & right optic neuritis and pale discs bilaterally & 2 & $20 / 30$ & $20 / 20$ \\
\hline 21 & f & 2 & left optic neuritis & 1 & $20 / 15$ & $20 / 20$ \\
\hline 47 & f & 3 & pale discs bilaterally & 11 & $20 / 30$ & $20 / 30$ \\
\hline 40 & f & 1 & left disc pallor & 2 & $20 / 20$ & $20 / 20$ \\
\hline 45 & m & 1 & episodes of bilateral vision loss and bilateral pale discs & 1 & $20 / 30$ & $20 / 20$ \\
\hline 37 & f & 1 & episodes of reduced vision left eye & 3 & $20 / 20$ & $20 / 30$ \\
\hline 32 & f & 2 & episodes of bilateral vision loss & 1 & $20 / 40$ & $20 / 60$ \\
\hline 53 & f & 3 & none & 7 & $20 / 20$ & $20 / 20$ \\
\hline 29 & f & 2 & none & 5 & $20 / 20$ & $20 / 30$ \\
\hline
\end{tabular}




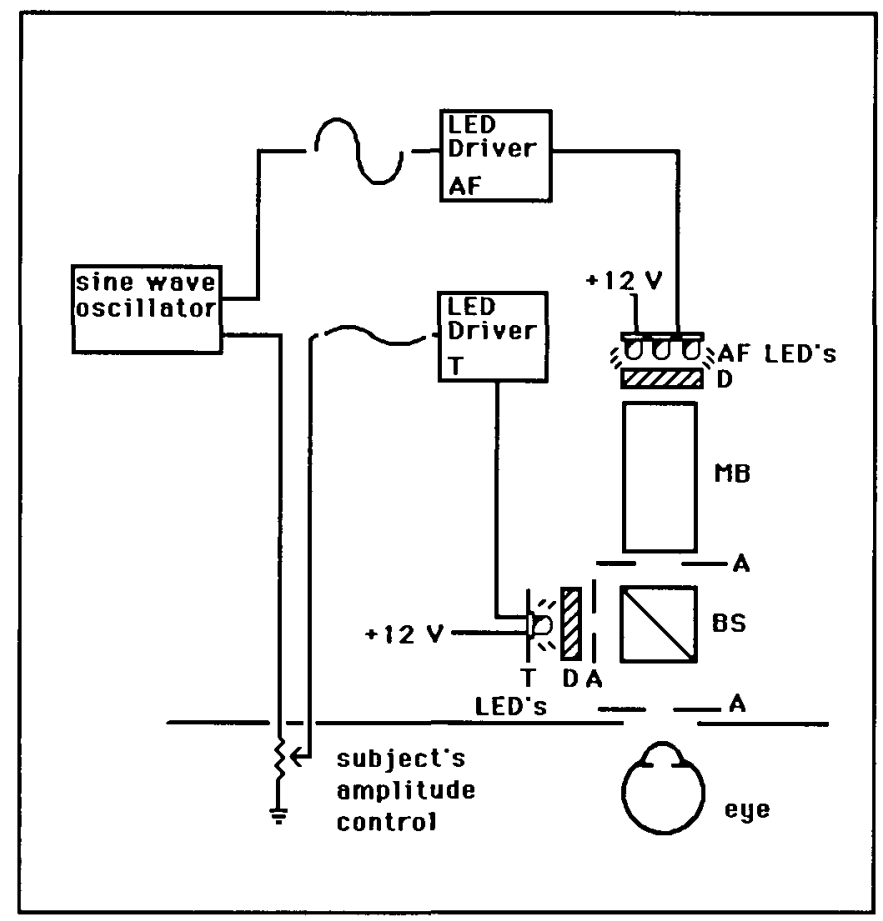

Figure I-A schematic of the apparatus. See text for details. AF, adapting field; $T$, test stimulus; $L E D$, light emitting diode; $D$, diffuser; $M B$, mirror box; BS, beam splitter.

nance of the adapting field was sinusoidally temporally modulated at a frequency of $7 \mathrm{~Hz}$ with a luminance modulation depth of $92 \%$. Modulation depth is defined as $\left[\left(L_{\max }-\mathrm{L}_{\min }\right) /\left(\mathrm{L}_{\max }+\right.\right.$ $\left.\left.L_{\min }\right)\right] X 100 \%$ where $L_{\max }$ in the maximal luminance of the display and $\mathrm{L}_{\min }$ is the minimal luminance. Modulation depth was measured with a photomultiplier. The time average luminance of this display (in the presence or absence of modulation) was $14 \mathrm{~cd} / \mathrm{m}^{2}$ as measured with an SEI photometer.

A single red LED, positioned at a right angle to the mirror box, was used to generate a 0.5 deg circular test stimulus. With the aid of a small beam splitter, the test spot appeared to the subject to be centered on the adapting field. A diagram of the apparatus is shown in Figure 1. A small test spot was used so that flicker measurements were not contaminated by edge effects. ${ }^{22}$ Apertures limiting the size of the test and adapting stimuli were placed at an equal distance from the observer so that no shift in accommodation was required upon presentation of the test stimulus. The mean luminance of the test spot was 27 $\mathrm{cd} / \mathrm{m}^{2}$. For all modulations, the test spot was always visible against the adapting field. The subject's task in the experiment was to adjust the modulation depth in the flicker of the test spot so that flicker was just detectable. This was done by turning the knob on a potentiometer. The range over which the potentiometer operated was varied randomly between threshold estimations so that the subject could not use the position of the knob as a cue. Subjects were informed of this at the beginning of the experiment.

The subject monocularly viewed the stimuli through a 14 $\mathrm{mm}$ aperture in a screen placed between the subject and the equipment. A chin rest was used to stabilize head position. The unused eye was occluded with an eye patch. The experiments were conducted in a darkened room. All subjects wore their own spectacles during the task when required.

\section{General Procedure}

In each experiment, four trials were conducted for each eye of each subject. The eye used was alternated after every second trial. A single experimental trial consisted of a $4.5 \mathrm{~min}$ period of viewing the adapting field. This period was interrupted every 15 seconds with a $5 \mathrm{sec}$ long presentation of the test stimulus. Whenever the test stimulus was present, the adapting field remained unmodulated to minimize edge contrast. At the onset of a test interval, the modulation depth in the test stimulus was set to a subthreshold value. The subject then adjusted the depth of modulation in the test spot until flicker was just barely detectable and then signalled the experimenter. (Note that unlike the measurement of critical flicker fusion, the frequency of flicker in the test spot was never varied.) Subjects experienced no difficulty in determining thresholds in the interval provided. At the beginning of each session, subjects were given verbal instructions regarding the task and two practice trials were conducted.

\section{Experiment I: Flicker Perception In The Absence Of FLicker AdaPtation}

The first experiment was conducted to assess flicker perception abnormalities during prolonged viewing of an unmodulated field. Wright et al." have reported that some MS patients have higher unadapted flicker modulation depth thresholds than healthy controls. Therefore, before the effects of flicker adaptation could be assessed, deficits in unadapted flicker thresholds were determined. Using the procedure described above, flicker thresholds were assessed every 15 seconds while subjects viewed an unmodulated, spatially uniform adapting field. Six patients participated in the experiments as did five of the healthy control subjects. Of the 12 patient eyes in this subset, 3 had no sign of symptom of visual involvement.

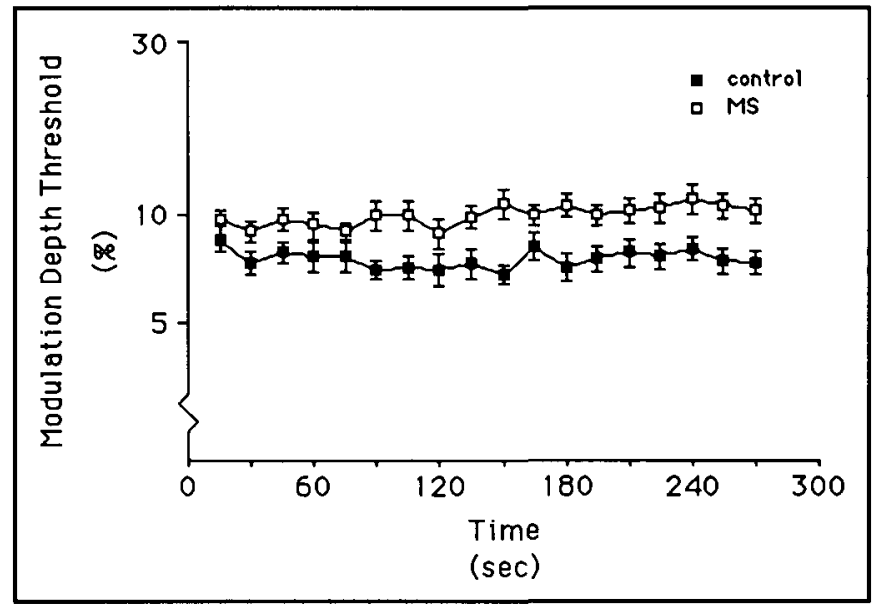

Figure 2 - Minimum modulation depth needed to just detect flicker in 0 small circular test stimulus as a function of time viewing an unmodulated adapting field for $M S$ and control groups. Open symbols repre sent group means for the MS patients and closed symbols represent group means for the control subjects. Vertical line represent $\pm I$ s.e. of the mean. 


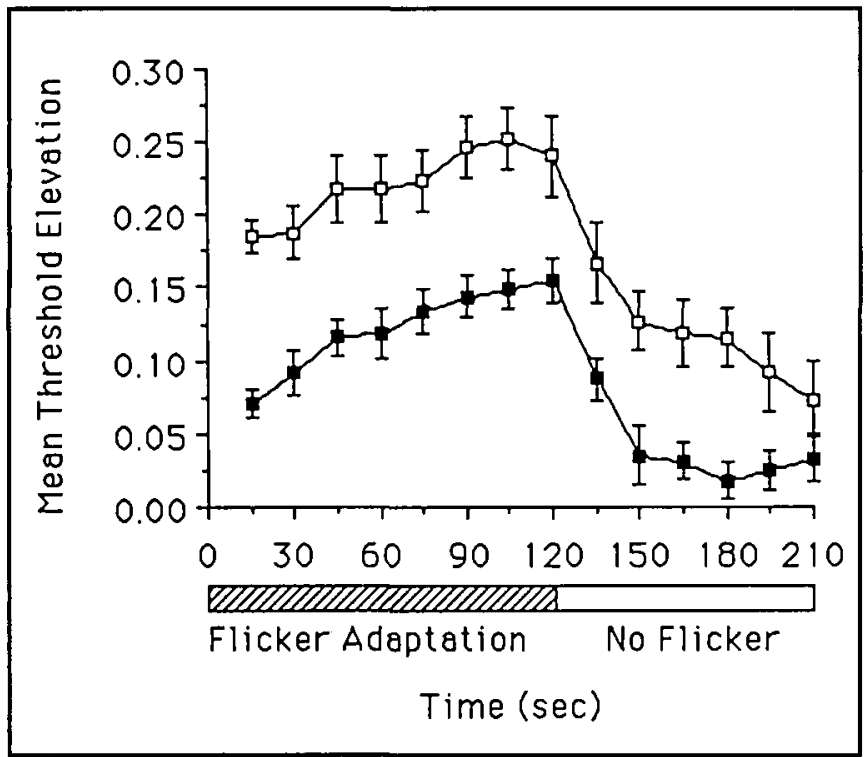

Figure 3-Group mean threshold elevation (i.e., log adapted thresholdlog baseline threshold) is plotted as a function of time viewing an adapting field. The adapting field was highly modulated for the first $120 \mathrm{sec}$ and thereafter remained unmodulated. Open symbols repre sent data from the $M S$ group and closed symbols represent data from the control group. Vertical lines represent \pm 1 s.e. of the mean.

\section{Results}

The group mean modulation depth thresholds obtained during Experiment I are shown in Figure 2. Modulation depth thresholds for the healthy control group did not vary significantly as a function of time of testing and averaged $7.2 \%$. Since the visual system typically performs a logarithmic transformation of sensory stimuli, statistical analyses for this and Experiment II were performed on log flicker thresholds. An analysis of variance on the mean log thresholds obtained from each eye showed that MS patients had slightly but significantly higher modulation depth thresholds $[F(1,323)=8.93, p<0.01]$ with an average value of $9.6 \%$. The analysis also showed that, unlike controls, MS patients' thresholds rose significantly with repeated testing $[F(17,323)=1.73$, p. $<0.05]$. MS thresholds became significantly different from control thresholds after 75 secs of viewing the unmodulated adapting field.

In a typical psychophysical experiment in which there is no visual adaptation (e.g., 19), threshold estimates are made within at least 60 seconds of viewing the stimulus. The average threshold obtained over the first 60 seconds of viewing for each eye in the present study was compared to the control average. A $99 \%$ confidence interval (using s.d. values) was calculated on the control group and thresholds from each patient eye were compared to these limits. It was found that none of the 3 unaffected eyes appeared abnormal using this criterion. Only four of the 9 affected eyes appeared abnormal, thus indicating that with short exposures to the stimulus, visual flicker deficits are not prevalent among MS patient eyes. When the last four thresholds are examined (after 3.5 minutes of viewing the uniform adapting disk), one of the 3 unaffected eyes appeared abnormal and 6 of the 9 affected eyes appeared abnormal. Thus, prolonged exposure to the unmodulated adapting field produced a sensitivity loss in some patients.

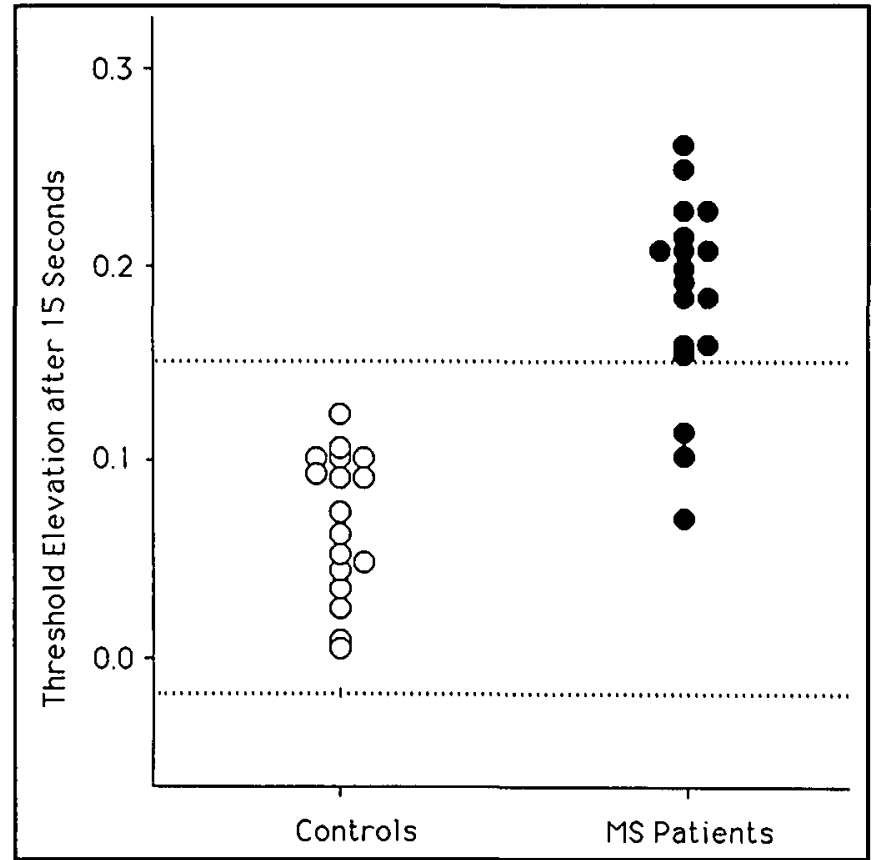

Figure 4 - Threshold elevation for individual eyes measured after 15 secs of adaptation to the highly modulated adapting field. Open symbols represent data from MS patients and closed symbols represent data from control subjects. The dashed horizonal lines represent \pm 2.33 s.d of the control group mean.

\section{EXPERIMENT II: ADAPTATION TO FliCKER}

Since the previous experiment indicated that MS patients' initial modulation depth threshold may be higher than those of controls, a within-subject design was chosen for flicker adaptation measurement. A within-subject design in which subjects' responses in a pre-adaptation condition were compared to their own responses in a post adaptation condition was also used to eliminate effects due to differences in pupil size (the level of ambient illumination was constant for both conditions) that may have existed between controls and MS patients. MS patients 1 to 9 participated in the experiment as did all the control subjects. The same basic procedure as above was used. As before, the first four threshold estimates were determined after viewing an unmodulated flickering adapting field. The next eight thresholds (i.e., adapted thresholds) were obtained after viewing a flickering adapting field. The last six thresholds (i.e. decay thresholds) were measured after viewing an unmodulated adapting field. The mean of the first four values was used as the baseline flicker threshold against which adapted and decay thresholds were compared. Threshold elevations due to adaptation are expressed as a difference in the log baseline threshold and the $\log$ adapted threshold. (Threshold elevation $=\log$ adapted threshold - log baseline threshold).

\section{Results}

Figure 3 illustrates the group mean changes in log flicker thresholds during and after adaptation to highly modulated flicker. An analysis of variance on the adapted threshold elevations showed a significant difference in the magnitude of the adaptation effects between the two subject groups $[\mathrm{F}(1,245)=$ $29.8, p<0.001]$. Both groups showed a significant increase in 
threshold elevation with prolonged viewing of the adapting field $[F(7,245)=10.1, p<0.001]$. The interaction effect of group and viewing time was not significant indicating that the rate of development of adaptation effects did not differ after the initial $15 \mathrm{sec}$ exposure to the adapting stimulus. However, the data clearly show that during the first $15 \mathrm{sec}$ of exposure, the buildup of adaptation effects was dramatically faster in MS patients than in controls.

One notable characteristic of the data was that the standard deviations of individual threshold estimates measured in MS patients were significantly $(p<0.01)$ greater than those for control subjects. A similar result was reported by Patterson, Foster and Heron ${ }^{23}$ who found that contrast detection threshold variability was abnormally high in MS patients relative to healthy controls during exposure to backgrounds of high luminance levels. This variability may be related to the "fading" of stimuli during adaptation intervals reported by some patients. In all cases patients reported that fading disappeared with the onset of the test stimulus and that it did not interfere with threshold adjustments. Fading was typically reported after at least 2 minutes into a testing trial.

The most consistent and dramatic effects of adaptation in the MS patients were seen after only 15 seconds of adaptation. At this early stage of adaptation, fading was never reported by patients and adaptation effects are relatively uncontaminated by nonspecific light adaptation shown in the previous experiment to occur in some MS patients after longer periods of viewing. The average threshold elevation found in the control group at this interval was $0.066 \log$ units (s.d. $=0.036$ ). Using s.d. in the calculation of confidence intervals, it was found that 5 of the 7 unaffected eyes and 10 of the 11 affected eyes in the MS group showed an average threshold elevation greater than the upper $99 \%$ confidence limit of the control group mean. All 9 MS patients could be distinguished from normal on this basis. Figure 4 shows the distribution of threshold elevations obtained from both groups.

All of the control eyes appeared normal.

The average threshold elevation measured in the control group during the entire adaptation period was $0.122 \log$ units (s.d. =0.035). Using s.d. in the calculation of confidence intervals, it was found that 3 of the 7 unaffected eyes in the MS group showed an average threshold elevation greater than the $99 \%$ confidence limits of the control group mean. Six of the 11 affected eyes had average values that appeared abnormal using this criterion. None of the control eyes appeared abnormal. Large variance in patient thresholds during adaptation, particularly in the latter half of the adaptation period may account for why some patients appeared abnormal initially in the adaptation period but had normal adaptation averages.

Following extinction of the flicker in the adapting field, modulation depth thresholds of controls subjects fell to preadaptation values within approximately 30 seconds. MS patients' thresholds remained elevated for a longer period of time, reaching near baseline values about 90 seconds after flicker adaptation had ceased.

\section{Discussion}

Prolonged viewing of a highly modulated flickering field produced transient losses in flicker modulation depth sensitivity for both MS patients and for healthy control subjects. Flicker adaptation effects for healthy subjects similar to those described here have been previously reported. 19.24 The present study has shown that both the magnitude of sensitivity loss and the rate with which these changes in flicker threshold are acquired as a consequence of flicker adaptation were significantly greater for MS patients than for healthy control subjects.

Physiological studies using experimental demyelination in animals have found that repeated electrical stimulation of partially demyelinated central nervous system neurons tended to increase the probability of complete axonal conduction blockade, thus enhancing the functional abnormalities already present in such neurons. ${ }^{16.17}$ Repeated stimulation of demyelinated fibres also caused them to fatigue more quickly and to a greater extent than fully myelinated neurons. These responses to extended stimulation suggest that temporary exacerbation of the effects of demyelination of visual structures in the brain of MS patients may be produced by prolonged visual stimulation. Such a process may account for the abnormally rapid and unusually large loss in flicker sensitivity observed in MS patients after flicker adaptation.

Prior to flicker or non-specific light adaptation, only 22 percent of the MS patients' eyes exhibited abnormal flicker sensitivity. In all cases, signs and symptoms of visual involvement were already present in these eyes. An initial control experiment in which subjects viewed an unmodulated adapting field, revealed that some patients and no controls experience a small loss in flicker sensitivity after viewing an unmodulated field for 2 to 3 minutes. Enoch et al. 25 observed that when patients viewed an intense, uniformly luminous field for a similar period of time, large losses in grating acuity could be temporarily produced. Both these data and those from the present study suggest that for MS patients, non-specific adaptation to light may cause reductions in sensitivity for a wide variety of visual functions. However, in the present study this effect was found for only $39 \%$ of the eyes tested and revealed a deficit in only 1 of the 7 eyes previously found to be uninvolved.

An important clinical contribution of a psychophysical analysis of visual function in MS patients could be to supplement information obtained from fundoscopic examination, visual acuity tests and patient reports of blurring and reduced vision episodes. Procedures which can identify clinically "normal" eyes as abnormal have potential use in the diagnostic process. Although tests of flicker sensitivity without adaptation or with nonspecific adaptation seem unable to detect subtle visual deficits, the present study shows that specific, i.e., flicker, adaptation procedures can produce temporary symptoms in otherwise symptomatic eyes, thus revealing subclinical visual involvement of the disease. After only 15 seconds of adaptation, 15 of the 18 eyes studied ( $83 \%$ ) demonstrated an abnormal sensitivity to flicker. Particularly noteworthy is that this procedure was able to identify 5 of the 7 unaffected eyes as abnormal. Contrast sensitivity (CS) measurements were available for 4 of the 5 eyes so identified and, in all cases, CS was found to be normal. Double flash data was available for 3 of the 5 eyes and, again, in all cases was found to be normal. Of the twelve eyes in the study for which double flash thresholds were available, 6 of the 6 eyes found to be normal with this test were observed to be abnormal with the flicker adaptation test. While the present pro- 
cedure may have significant diagnostic value, additional research is needed to determine how specific the observed effects are for MS as opposed to other neurological and ophthalmological disorders.

The results of the present experiment suggest that partial demyelination of visual pathway neurons may exist in MS patients without signs or symptoms of visual involvement. The prolonged stimulation provided during visual adaptation procedures may produce sufficient exacerbation of neural transmission abnormalities to render reductions in sensory sensitivity. Unlike other methods used clinically to produce temporary exacerbation of symptoms in MS for the purpose of diagnosis (e.g., hot bath or exercise), visual adaptation is local to the visual system, painless, and recovery is complete within a few minutes.

\section{ACKNOWLEDGEMENTS}

This work was funded by a grant to J. Raymond from the Alberta Heritage Foundation for Medical Research.

\section{REFERENCES}

1. Lumsden CE. The neuropathology of multiple sclerosis. In: Handbook of Clinical Neurology, volume 9, Vinken PJ and Bruyn GW, eds. Amsterdam, North Holland Publishing, 1970.

2. Ikuta $F$ and Zimmerman HM. Distribution of plaques in seventy autopsy cases of MS in the United States. Neurologica 1976; 26: 27.

3. Kurtzke JF. Clinical manifestations of multiple sclerosis. In: Handbook of Clinical Neurology, volume 9, Vinken PJ and Bruyn GW, eds. Amsterdam, North Holland Publishing, 1970.

4. Galvin RJ, Regan D and Heron JR. Impaired temporal resolution of vision after acute retrobulbar neuritis. Brain 1976; 99: 255-268.

5. Brussel EM, White CW, Bross M, et al. Multi-flash campimetry in multiple sclerosis. Curr Eye Res 1981; 1: 671-677.

6. Overbury O, Brussel EM, White CW, et al. Evaluating loss with multi-flash campimetry. Can J Ophthalmol 1984; 19: 255-260.

7. Parsons OA and Miller PN. Flicker fusion thresholds in multiple sclerosis. Arch Neurol 1957; 77: 134-139.

8. Daley ML, Swank RL and Ellison CM. Flicker fusion thresholds in multiple sclerosis: a functional measure of neurological damage. Arch Neurol 1979; 36: 292-295.

9. Rushton D. Use of the Pulfrich pendulum for detecting abnormal delay in the visual pathway in multiple sclerosis. Brain 1975; 98: 283-296.
10. Heron JR, Regan D and Milner BA. Delay in visual perception in unilateral optic atrophy after retrobulbar neuritis. Brain 1974: 97: 69-78.

11. Wright CE, Drasdo $\mathrm{N}$ and Harding GFA. Pathology of the optic nerve and visual association areas: Information given by the flash and pattern visual evoked potential, and the temporal and spatial contrast sensitivity function. Brain 1987; 110: 107-120.

12. Hess RF and Plant GT. The effect of temporal frequency variation on threshold contrast sensitivity deficits in optic neuritis. J Neurol Neurosurg and Psychiatry 1983; 46: 322-330.

13. Plant GT and Hess RF. Temporal frequency discrimination in optic neuritis and multiple sclerosis. Brain 1985; 108: 647-676.

14. Galvin RJ, Regan D and Heron JR. A possible means of monitoring the progress of demyelination in multiple sclerosis. J Neurol 1976; 39: 861-865.

15. Sekuler R, Owsley, C and Berenberg R. Contrast sensitivity during provoked visual impairment in multiple sclerosis. Ophthalmic Physiol Opt 1986; 6: 229-232.

16. McDonald WI and Sears TA. The effects of experimental demyelination on conduction in the central nervous system. Brain 1970; 93: 583-598.

17. Davis FA. Impairment of repetitive impulse conduction in experimentally demyelinated and pressure injured nerves. J Neurol Neurosurg and Psychiatry 1972; 35: 537-544.

18. Blakemore $\mathrm{C}$ and Campbell FW. On the existence in the human visual system of neurons selectively sensitive to the orientation and size of retinal images. J Physiol 1969; 203: 237-260.

19. Smith RA. Studies of temporal frequency adaptation in visual contrast sensitivity. J Physiol 1971; 216: 531-552.

20. Schumacher GA, JBeebe $G$ and Kilber RE. Problems of experimental trials of therapy in multiple sclerosis: report of the panel on evaluation of experimental trial in multiple sclerosis. Ann NY Acad Sci 1968; 122: 522-568.

21. Kurtzke JF. Further notes on disability evaluation in multiple sclerosis, with scale modifications. Neurology 1965; 15: 654-661.

22. Kelly DH. Visual responses to time-dependent stimuli. 1 Amplitude sensitivity measurements. J Opt Soc Am 1961; 51: 422-429.

23. Patterson VH, Foster DH and Heron JR. Variability of visual threshold in multiple sclerosis: Effect of background luminance on frequency of seeing. Brain 1980; 103: 139-147.

24. Regan D and Beverley KI. Looming detectors in the human visual pathway. Vis Res 1978; 18: 415-421.

25. Enoch JM, Campos EC and Bedell HE. Visual resolution in a patient exhibiting visual fatigue or saturation-like effect. Arch Ophthalmol 1979; 97: 76-78. 